\title{
Achados audiológicos em indivíduos com distrofia miotônica de Steinert
}

\author{
Audiologic findings in individuals with Steinert's myotonic dystrophy
}

\author{
Lorene Lemos Lins ${ }^{1}$, Carla Marcondes César Affonso Padovani², Rita Lucena ${ }^{3}$, Lorena Silva de Argolo ${ }^{4}$, \\ Luciene Cruz Fernandes ${ }^{5}$, Camila Vila-Nova de Freitas Guimarães ${ }^{6}$, Fernanda Costa de Queirós ${ }^{7}$
}

\begin{abstract}
RESUMO
Objetivo: Identificar as principais alterações audiológicas em indivíduos portadores de distrofia miotônica de Steinert. Métodos: Cinco indivíduos foram submetidos à audiometria tonal e vocal, imitanciometria, teste de função tubária, pesquisa do reflexo estapediano, declínio do reflexo acústico e aplicação de dois questionários: a) Desempenho da função auditiva; b) Investigação psico-acústica-social. Resultados: $\mathrm{O}$ estudo demonstrou que $60 \%$ da amostra apresentaram limiares auditivos dentro dos padrões de normalidade, $10 \%$ perda condutiva e 30\% perda auditiva nas freqüências altas. Em relação aos reflexos estapedianos, observou-se o percentual de alteração de $30 \%$ nas freqüências de 0,5 e 1 kHz e de $40 \%$ em 2 e 4 kHz. Observou-se declínio do reflexo acústico em $10 \%$ da amostra nas frequiências de 0,5 e 1 kHz. Conclusão: O estudo mostrou que o padrão audiológico típico de perda auditiva em frequiências altas foi observado em $30 \%$ da amostra.
\end{abstract}

Descritores: Distrofia miotônica/fisiopatologia; Distrofia miotônica/complicações; Perda auditiva; Audição; Testes de impedância acústica; Estapédio; Tuba auditiva; Reflexo/genética

\section{INTRODUÇÃO}

A distrofia miotônica, também conhecida por doença de Steinert ou miotonia atrófica foi descrita como entidade clínica em 1909, por Steinert ${ }^{(1)}$.

A distrofia muscular de Steinert (DMS) é uma doença multissistêmica autossômica dominante, de expressão variável, caracterizada pela expansão de trinucleotídeos - CTG, na região 3" do gene da quinase protéica, no cromossomo $19^{(2)}$.

As primeiras manifestações afetam preferencialmente os músculos esqueléticos, sendo a característica clínica mais relevante uma debilidade muscular progressiva, atrofia muscular

Trabalho realizado na Universidade do Estado da Bahia - UNEB - Salvador (BA), Brasil, com bolsa PIBIC, processo número 800010/96-5.

(1) Fonoaudióloga do Centro de Atendimento Fonoaudiológico do Hospital Santo Antonio - Obras Sociais de Irmã Dulce - Salvador (BA), Brasil.

(2) Doutora, Professora Adjunto do Curso de Fonoaudiologia da Universidade do Estado da Bahia - UNEB - Salvador (BA), Brasil.

(3) Doutora, Professora do Programa de Pós Graduação em Medicina e Saúde da Universidade Federal da Bahia - UFBA - Salvador (BA), Brasil.

(4) Fonoaudióloga do Centro de Atendimento Fonoaudiológico do Hospital Santo Antonio - Obras Sociais de Irmã Dulce - Salvador (BA), Brasil.

(5) Mestre, Professora Assistente do Curso de Fonoaudiologia da Universidade do Estado da Bahia - UNEB - Salvador (BA), Brasil.

(6) Mestre, Professora do Curso de Fonoaudiologia da União Metropolitana de Educação e Cultura - UNIME - Lauro de Freitas (BA), Brasil.

(7) Mestre, Professora do Curso de Fonoaudiologia da União Metropolitana de Educação e Cultura - UNIME - Lauro de Freitas (BA), Brasil.

Endereço para correspondência: Lorene Lemos Lins. R. Professor Alfredo Rocha, 06/104, Vila Laura, Salvador - BA. CEP 40270-150. E-mail: lorene_lins@yahoo.com.br

Recebido em: 21/11/2007; Aceito em: 7/9/2008 e fenômenos miotônicos, entendidos como uma dificuldade de relaxamento do músculo após contração ${ }^{(1)}$.

A distrofia miotônica subdivide-se em quatro formas clínicas: congênita, infantil, clássica e leve. Na distrofia miotônica congênita, durante o período pré-natal, ocorre diminuição da movimentação do feto. Ao nascer, a criança apresenta dificuldades respiratórias, de sucção e deglutição, decorrentes da hipotonia e da fraqueza muscular. Na forma infantil, a hipotonia e fraqueza são generalizadas e os fenômenos miotônicos são perceptíveis por volta de cinco a dez anos de idade ${ }^{(1)}$.

A distrofia miotônica clássica ocorre na fase adulta e é associada a fenômenos miotônicos; com presença de alterações cardíacas, catarata, disfagia, disartria, alterações na qualidade vocal - devido ao comprometimento de musculatura faríngea e laríngea - e oftalmoparesia. Na forma leve, o comprometimento muscular é discreto; com presença de miotonia e fraqueza muscular sem implicações significativas para os indivíduos portadores da doença ${ }^{(1)}$.

Quanto aos aspectos fonoaudiológicos, os portadores de DMS apresentam alteração da mímica facial, ptose palpebral, atrofia de músculos mastigatórios, fraqueza na musculatura orofaríngea e laríngea, comprometimento da fala e alteração vocal. Em relação aos achados audiológicos, há evidências de perda auditiva em freqüências altas ${ }^{(3-4)}$.

Um estudo realizado com 13 portadores de DMS concluiu que os tempos de contração e de relaxamento do músculo estapédio apresentaram-se mais prolongados, supondo uma correlação direta com a fraqueza muscular e miotonia presentes nesses indivíduos ${ }^{(5)}$, o que corrobora com dados de outros autores que também afirmaram haver prolongamento do 
tempo de relaxamento do músculo estapediano em portadores de $\operatorname{DMS}^{(6-7)}$.

Uma das funções desse músculo é o efeito de anti-mascaramento, definido como a atenuação de frequiências baixas de sons ambientais ou oriundos do próprio indivíduo. Desta forma, a contração do músculo estapédio durante a fala reduz o mascaramento causado pelas baixas freqüências, o que melhora a percepção das freqüências altas, proporcionando aumento da inteligibilidade da fala ${ }^{(8-11)}$. Assim, a função do reflexo estapediano como efeito anti-mascarante pode estar prejudicada caso haja alteração desse músculo nos casos de distrofia muscular de Steinert.

A alteração do reflexo estapediano nos indivíduos com DMS poderia gerar prejuízos, no processamento auditivo central, devido ao efeito anti-mascarante. Um estudo avaliou o desempenho no reconhecimento de dissílabos em tarefa dicótica em sujeitos com audição normal e reflexos estapedianos ausentes. Percebeu-se que os indivíduos com limiares de reflexo estapediano elevados ou ausentes podem apresentar alterações nas habilidades auditivas, relacionadas ao processamento auditivo central e, conseqüentemente, queixas quanto à inteligibilidade de fala ${ }^{(12)}$.

A ausência do reflexo estapediano, em orelhas que não apresentem comprometimento tímpano-ossicular, pode sugerir comprometimento do complexo olivar superior em disparar o comando neural do nervo facial na contração do músculo estapédio. O complexo olivar superior, além de exercer a função de controle sobre ação do nervo facial na contração do músculo estápedio, também gerencia as habilidades auditivas, na presença de ruído competitivo e na seletividade de frequiências. Portanto, por conta dessas duas últimas funções, a investigação do reflexo estapediano em indivíduos com queixas de inteligibilidade de fala, pode indicar alteração no processamento auditivo central, caso seja constatada ausência de reflexo ${ }^{(11)}$.

Pesquisadores realizaram um estudo com 13 portadores de DMS em que sete apresentaram perda auditiva neurossensorial em frequiências altas, não esperadas para idade, atribuída à distrofia muscular. Esse estudo sugeriu que esses sujeitos apresentam "presbiacusia precoce", sendo considerados idosos funcionais. Revelou, ainda, níveis de reflexo estapediano e timpanometria dentro dos padrões de normalidade ${ }^{(4)}$.

Anteriormente, outro estudo identificou perda auditiva neurossensorial de grau moderado a severo em 17 dos 25 sujeitos avaliados com $\mathrm{DMS}^{(13)}$.

Um número significativo de indivíduos com DMS apresentam perda auditiva em frequiências altas, com a possibilidade de envolvimento coclear e retrococlear, porém sem envolvimento das freqüências de fala. Algumas anomalias ósseas têm sido descritas na distrofia miotônica, incluindo alterações no osso temporal que poderão comprometer o canal auditivo interno $^{(14)}$.

Essa pesquisa resultou do aprofundamento acerca do conhecimento sobre DMS e da preocupação em avaliar os músculos relacionados ao sistema auditivo, visto que essa enfermidade é caracterizada por um quadro de comprometimento muscular progressivo.

Sendo assim, esse estudo teve como objetivo identificar as principais alterações audiológicas em indivíduos portadores de DMS. Buscou-se: a) Avaliar a efetividade de contração do músculo estapédio; b) Investigar as repercussões psico-acústica-sociais em portadores de DMS; c) Pesquisar a ocorrência de alteração da função da tuba auditiva.

O estudo pormenorizado dos aspectos audiológicos nesses pacientes torna-se relevante para intervenção fonoaudiológica mais adequada, buscando melhora na qualidade de vida de portadores de distrofia muscular de Steinert.

\section{MÉTODOS}

Realizou-se um estudo de série de casos no serviço de Neurociências de um hospital de referência localizado na cidade de Salvador (BA).

Foram selecionados aleatoriamente 11 indivíduos, faixa etária entre 28 a 50 anos, atendidos no setor de doenças neuromusculares em 2004. Desses, quatro não aceitaram participar da pesquisa e dois não atendiam aos critérios de inclusão da pesquisa. Assim, somente cinco indivíduos foram elegíveis para o estudo. As avaliações foram realizadas em uma clínica escola de Fonoaudiologia, situada na cidade de Salvador (BA). O contato inicial foi feito por telefone. Neste, agendou-se uma reunião com o intuito de apresentar os objetivos e as etapas da pesquisa, bem como assinar o termo de consentimento livre e esclarecido para aqueles que aceitassem participar do estudo (Pesquisa aprovada pelo Comitê de Ética e Pesquisa da Universidade do Estado da Bahia, que dispõe da resolução 196/96).

O critério de inclusão foi o diagnóstico fenotípico e eletrofisiológico da DMS. Os critérios de exclusão foram: a) distúrbios metabólicos; b) alterações audiológicas provenientes do uso de drogas e/ou ototóxicos; c) exposição prévia a ruídos; d) comprometimento neurológico concomitante a DMS; e) condições clínicas que inviabilizassem a realização dos exames (dificuldade de locomoção, alterações cardíacas ou pulmonares).

Aplicou-se uma anamnese específica (Anexo 1) para a coleta de informações relativas à possível presença de doenças metabólica ou infecciosa, diagnóstico da doença, tipo de fraqueza muscular.

Todos os sujeitos foram submetidos à avaliação audiológica composta de audiometria tonal limiar, logoaudiometria, teste de função tubária, pesquisa do declínio do reflexo estapediano e medidas da imitância acústica: timpanometria e pesquisa dos reflexos acústicos contralaterais nas freqüências de $0,5 \mathrm{kHz}$, $1 \mathrm{kHz}$ e $2 \mathrm{kHz}$. Foram utilizados os seguintes critérios para a avaliação do reflexo acústico:

a) Primeiramente, pesquisou-se os limiares nas freqüências de $0,5 \mathrm{kHz}, 1 \mathrm{kHz}, 2 \mathrm{kHz}$ e $4 \mathrm{kHz}$, respectivamente;

b) Iniciou-se na intensidade de $80 \mathrm{~dB}$ NA, de forma ascendente, com incrementos de $5 \mathrm{~dB}$ até que apresentasse uma variação na agulha maior ou igual a $0.03 \mathrm{ml}$, sendo assim, considerado o limiar do reflexo acústico (conforme manual do equipamento utilizado).

$\mathrm{Na}$ pesquisa do reflexo acústico, foram considerados normais os limiares menores ou iguais a $100 \mathrm{~dB}$ NA. Valores superiores foram considerados elevados ${ }^{(15)}$.

Foi realizada a audiometria vocal, pesquisando-se o limiar 
de reconhecimento de fala (LRF) e o índice de reconhecimento de fala (IRF). Para o LRF, após a determinação dos limiares tonais, foi utilizada intensidade confortável para o paciente de 30 a $40 \mathrm{~dB}$ acima da média tonal de $0,5,1$ e 2 $\mathrm{kHz}$, com a emissão das palavras, solicitando ao paciente que as repetisse. Falava-se uma palavra em cada instante e, em seguida, foi diminuída de $10 \mathrm{em} 10 \mathrm{~dB}$ até o instante em que o examinando não conseguisse mais repeti-las. Neste momento aumentava-se a intensidade de $5 \mathrm{em} 5 \mathrm{~dB}$, selecionando quatro palavras para cada intensidade. Considerou-se $50 \%$ de acerto na repetição das palavras apresentadas como o limiar de recepção de fala.

Em relação ao IRF, foram utilizadas listas de palavras monossílabas e dissílabas, balanceadas foneticamente. Para o início do teste, acrescentou-se $40 \mathrm{~dB}$ acima da média tonal de $0,5,1$ e $2 \mathrm{kHz}$ e solicitou-se ao paciente que repetisse as palavras ouvidas, sendo um total de 25 palavras para cada orelha. Como são 25 palavras, cada acerto representou $4 \%$ do total.

Os valores de referência para a audiometria tonal e vocal foram baseados naqueles descritos por Davis e Silverman*, citado por Katz ${ }^{(16)}$. Para a timpanometria, utilizaram-se valores de Jerger ${ }^{(17)}$ e para o reflexo estapediano a referência utilizada foi a de Jerger, Oliver e Chmiel ${ }^{(15)}$.

Utilizou-se o equipamento de marca Audiotest modelo 227 para realização da audiometria tonal e vocal, e o imitanciômetro modelo AZ7, para a realização dos demais exames.

No teste de função tubária, introduziu-se uma pressão positiva de $200 \mathrm{da} \mathrm{Pa}$, solicitando que o indivíduo deglutisse um volume de água, com o intuito de provocar a abertura ativa da tuba ativa pela contração do músculo tensor do palato. Solicitaram-se três deglutições. Consideramos a função normal, quando houve diminuição gradativa da pressão até 0 da Pa e alterada, quando não houve qualquer diminuição da pressão ou quando ocorreu uma queda abrupta da pressão.

Para a realização do tone decay, aplicou-se, durante 20 segundos, um tom puro nas frequiências de $0,5,1,2$ e $4 \mathrm{kHz}$, $10 \mathrm{~dB}$ acima do limiar do reflexo estapediano. Observou-se o movimento da agulha. Quando esta se manteve na mesma posição durante os 20 segundos, considerou-se tone decay negativo, se decaiu no mínimo $50 \%$ da posição inicial nos primeiros segundos, tone decay positivo.

Foram utilizados dois questionários elaborados pelos pesquisadores a partir dos instrumentos utilizados no local da pesquisa, a fim de avaliar: a) Desempenho da função auditiva (Anexo 2); b) Investigação psico-acústica-social (Anexo 3).

Os dados coletados foram analisados com base no programa estatístico SPSS for Windows (versão 10.0). Os gráficos foram elaborados no SPSS for Windows (versão 10.0) e no Microsoft Excel.

\section{RESULTADOS}

Os resultados foram apresentados de duas formas: em conjunto com cinco sujeitos avaliados e individualmente (Quadro
1). Os pesquisadores consideram essa forma de apresentação de dados mais favorável para embasar a discussão.

Houve o predomínio do gênero masculino (60\%) em relação ao feminino (40\%). Todos os avaliados referiram apresentar algum tipo de fraqueza muscular, sendo relatado $20 \%$ apenas em membros superiores e $80 \%$ em duas ou mais regiões musculares pesquisadas: membros inferiores e superiores, musculatura facial e/ou cervical.

Antes da realização dos exames audiológicos, todos os indivíduos foram submetidos à meatoscopia. Apenas um indivíduo foi encaminhado para consulta otorrinolaringológica para remoção de rolha de cerume.

De acordo com o questionário de desempenho da função auditiva, apenas $20 \%$ relataram queixa auditiva, não havendo alterações nos demais itens.

Em relação à investigação psico-acústica-social, $40 \%$ referiram presença de zumbido. Do total, $20 \%$ relataram o incômodo a sons de forte intensidade e $40 \%$ referiram dificuldade para entender conversas e presença de tontura ou vertigem.

As análises da pesquisa foram realizadas por orelha. Assim, da população estudada, $60 \%$ apresentaram limiares auditivos dentro dos padrões de normalidade; $10 \%$ perda condutiva e $30 \%$ perda auditiva nas frequiências altas. Constatou-se aumento de limiares, principalmente, nas freqüências de 6 e $8 \mathrm{kHz}$ (Figura 1).

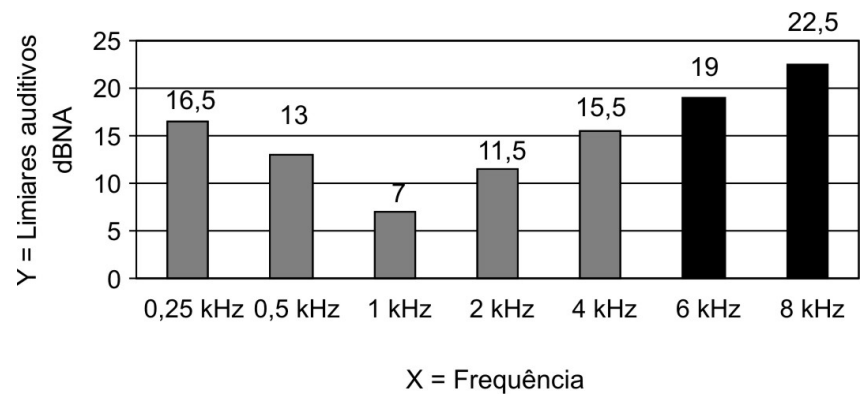

Figura 1. Média dos limiares auditivos dos indivíduos, em cada freqüência testada

Na observação da Figura 1, verificou-se que os limiares nas frequiências de 6 e $8 \mathrm{kHz}$ não excederam os $25 \mathrm{~dB}$ quando apresentados em conjunto. Contudo, na análise individual, observouse que os sujeitos $\mathrm{S} 4 \mathrm{e}$ S5 apresentaram limiares elevados nessas frequiências, que excederam os $25 \mathrm{~dB}(30-50 \mathrm{~dB})$.

Com relação aos resultados da audiometria tonal, foi realizada análise simultânea entre as variáveis $X$ (freqüências testadas) e Y (limiares auditivos dos pacientes), por meio de correlação linear simples. Observou-se uma correlação positiva entre elas. Primeiro, pela própria análise do gráfico. Segundo, pelo valor do coeficiente de correlação (r) encontrado. Quanto mais próximo de um, mais forte é a correlação. Na pesquisa, pode-se afirmar que a correlação entre as variáveis foi de moderada a forte, já que o $\mathrm{r}$ foi de 0,7711 (Figura 2).

Não foram encontradas alterações nos limiares vocais e/ ou incompatibilidade entre estes e os limiares tonais, quando observados em conjunto.

*Davis H, Silverman SR. Hearing and deafness. New York: Holt, Rinehart and Winston, Inc., 1970. 
Quadro 1. Resultados individuais das avaliações dos sujeitos

\begin{tabular}{|c|c|}
\hline $\begin{array}{l}\text { Sujeito 1: S1 } \\
\text { 1. Identificação: E.M.M.C. } \\
\text { 2. Sexo: masculino Idade: } 24 \\
\text { 3. Fraqueza muscular: membros inferiores } \\
\quad \text { musculatura facial } \\
\text { 4. Passado otológico: sem relato } \\
\text { 5. Queixa auditiva: nenhuma } \\
\text { 6. Audiometria tonal: } \\
\text { OD: limiares auditivos normais } \\
\text { OE: limiares auditivos normais } \\
\text { 7. Audiometria vocal: } \\
\text { OD: LRF: } 15 \mathrm{~dB} \text { IRF: } 100 \% 50 \mathrm{~dB} \\
\text { OE: LRF: } 5 \text { dB IRF: } 100 \% 45 \mathrm{~dB} \\
\text { 8. Timpanometria: } \\
\text { OD: curva tipo A } \\
\text { OE: curva tipo A } \\
\text { 9. Reflexo estapediano contralateral: } \\
\text { OD: presentes } \\
\text { OE: presentes }\end{array}$ & $\begin{array}{l}\text { Sujeito 2: S2 } \\
\text { 1. Identificação: S.M.R. } \\
\text { 2. Sexo: feminino Idade: } 28 \\
\text { 3. Fraqueza muscular: membros superiores } \\
\quad \text { membros inferiores } \\
\text { 4. Passado otológico: sem relato } \\
\text { 5. Queixa auditiva: dificuldade de entender conversas } \\
\text { 6. Audiometria tonal: } \\
\text { OD: limiares auditivos normais } \\
\text { OE: limiares auditivos normais } \\
\text { 7. Audiometria vocal: } \\
\text { OD: LRF: } 10 \mathrm{~dB} \text { IRF: } 100 \% 45 \mathrm{~dB} \\
\text { OE: LRF: } 10 \mathrm{~dB} \text { IRF: } 100 \% 50 \mathrm{~dB} \\
\text { 8. Timpanometria: } \\
\text { OD: curva tipo A } \\
\text { OE: curva tipo A } \\
\text { 9. Reflexo estapediano contralateral: } \\
\text { OD: presentes } \\
\text { OE: presentes }\end{array}$ \\
\hline $\begin{array}{l}\text { Sujeito 3: S3 } \\
\text { 1. Identificação: S.M.N. } \\
\text { 2. Sexo: feminino Idade: } 50 \\
\text { 3. Fraqueza muscular: membros superiores } \\
\text { 4. Passado otológico: sem relato } \\
\text { 5. Queixa auditiva: nenhuma } \\
\text { 6. Audiometria tonal: } \\
\text { OD: limiares auditivos normais } \\
\text { OE: limiares auditivos normais } \\
\text { 7. Audiometria vocal: } \\
\text { OD: LRF: } 15 \mathrm{~dB} \text { IRF: } 100 \% 55 \mathrm{~dB} \\
\text { OE: LRF: } 10 \mathrm{~dB} \text { IRF: } 100 \% 55 \mathrm{~dB} \\
\text { 8. Timpanometria: } \\
\text { OD: curva tipo A } \\
\text { OE: curva tipo A } \\
\text { 9. Reflexo estapediano contralateral: } \\
\text { OD: presentes em níveis elevados } \\
\text { OE: presentes em níveis elevados }\end{array}$ & 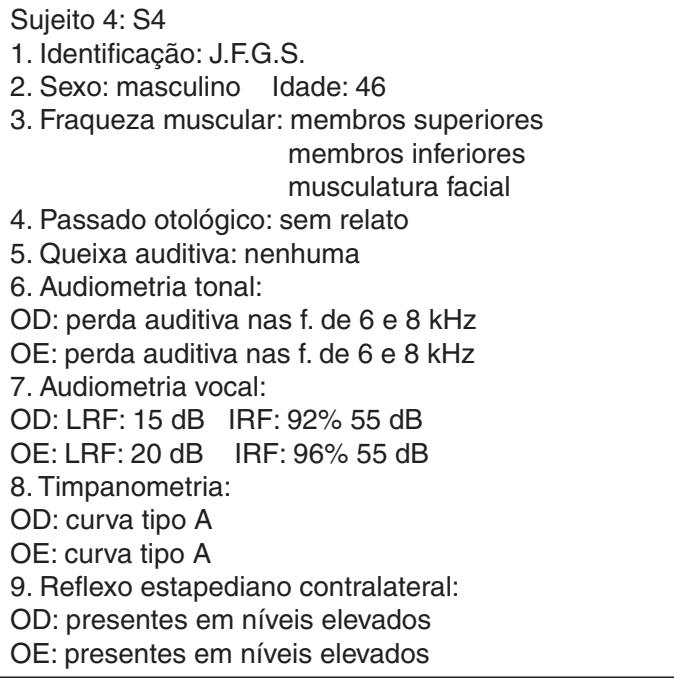 \\
\hline
\end{tabular}

Sujeito 5: S5

1. Identificação: A.E.A.

2. Sexo: masculino Idade: 36

3. Fraqueza muscular: membros superiores

4. Passado otológico: sem relato membros inferiores

5. Queixa auditiva: nenhuma

6. Audiometria tonal:

OD: perda auditiva condutiva

OE: perda auditiva nas f. de 6 e $8 \mathrm{kHz}$

7. Audiometria vocal:

OD: LRF: $10 \mathrm{~dB}$ IRF: $100 \% 55 \mathrm{~dB}$

OE: LRF: $20 \mathrm{~dB}$ IRF: $100 \% 50 \mathrm{~dB}$

8. Timpanometria:

OD: curva tipo $\mathrm{C}$

OE: curva tipo A

9. Reflexo estapediano contralateral:

OD: presentes em níveis elevados

OE: presentes em níveis elevados

Em relação à timpanometria, 90\% dos sujeitos apresentaram curva timpanométrica do tipo A e 10\% curva timpanométrica do tipo $\mathrm{C}$.

Não houve alteração nos testes da função tubária.

Em relação aos reflexos estapedianos, observou-se percentual de alteração de $30 \%$ nas freqüências de 0,5 e $1 \mathrm{kHz}$, sendo $10 \%$ ausentes e $20 \%$ limiares elevados. Na freqüência de $2 \mathrm{kHz}$, o percentual de alteração foi de $40 \%$, sendo $20 \%$ ausente e $20 \%$ em limiares elevados. Na frequiência de $4 \mathrm{kHz}$, os reflexos apresentaram-se ausentes em $40 \%$ da amostra (Figura 3).

Observou-se nas frequiências de 0,5 e $1 \mathrm{kHz}$ que apenas $10 \%$ apresentaram declínio do reflexo acústico e em 10\%, não foi possível realizar a avaliação, pois os limiares do reflexo estapediano estavam ausentes ou elevados. Na freqüência de 2 e 4 kHz não houve alteração. 


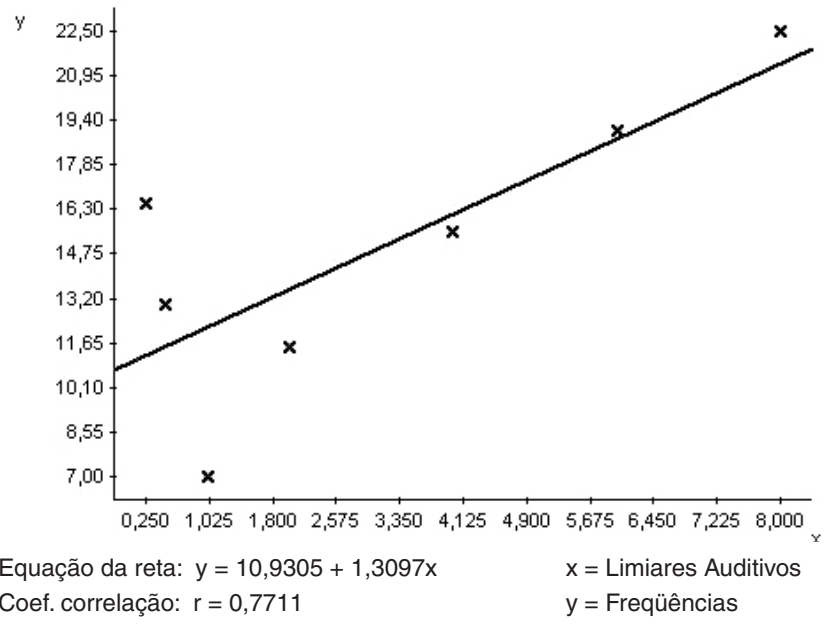

Figura 2. Regressão positiva entre limiares auditivos e freqüências testadas

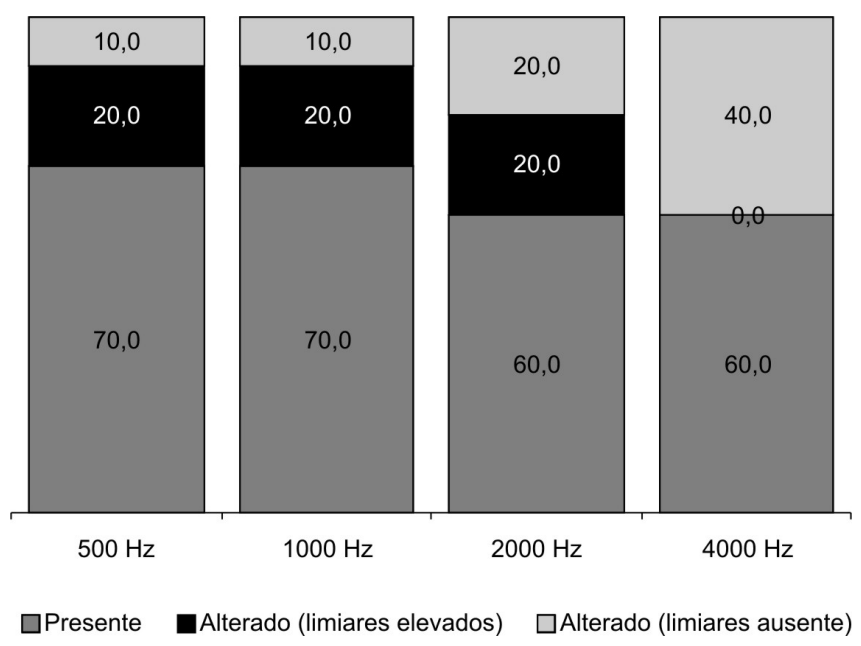

Figura 3. Ocorrência do reflexo estapediano

\section{DISCUSSÃO}

Todos os sujeitos avaliados referiram apresentar algum tipo de fraqueza muscular. Observou-se que os participantes com maior tempo de doença, apresentavam mais regiões musculares comprometidas. Atribui-se a esse resultado o fato de que a DMS é uma doença muscular progressiva.

Em relação aos sujeitos S4 e S5, pode-se fazer uma correlação positiva entre a idade, a evolução clínica da doença e, conseqüentemente, a elevação dos limiares nas freqüências de 6 e $8 \mathrm{kHz}$.

O perfil audiológico de $30 \%$ da população estudada foi condizente com os achados da literatura de perda auditiva nas freqüências altas ${ }^{(4,13-14)}$.

Do total estudado, 30\% apresentaram perda auditiva nas freqüências altas na faixa etária de 35 a 45 anos, representados individualmente pelos sujeitos S4 e S5. Neste caso, pode-se considerar a ocorrência de "presbiacusia precoce", visto que essa configuração de perda não é esperada para essa faixa etária. A configuração da alteração pode, então, ser atribuída à distrofia muscular e ao quadro de fraqueza em geral ${ }^{(4)}$.
Os sujeitos S4 e S5 apresentaram correlação positiva entre elevação dos limiares tonais nas freqüências altas e evolução clínica do quadro pesquisado. Esses indivíduos, além de apresentarem idade superior a 35 anos, relataram mais regiões musculares comprometidas.

Ainda, em relação aos achados dos limiares tonais, hipotetizou-se que os sujeitos da pesquisa em questão podem ser portadores de alguma lesão óssea, que justificaria a perda auditiva neurossensorial, já que um estudo ${ }^{(14)}$ descreveu a ocorrência de alterações ósseas em indivíduos com DMS, com envolvimento do osso temporal, ocasionando lesão coclear ou retrococlear.

Assim, a ocorrência de perda auditiva neurossensorial em frequiências altas observada na pesquisa em questão teria duas possibilidades: fraqueza do músculo estapediano e possível anomalia óssea.

Em relação à investigação psico-acústica-social, 40\% referiram zumbido, presença de tontura e vertigem, e dificuldade na compreensão auditiva durante situações de diálogo. Apenas $20 \%$ relataram o incômodo a sons de forte intensidade.

Observou-se limiares de reflexo acústico alterados, classificados em ausentes ou em níveis elevados. Estes achados, junto ao histórico otológico negativo, contribuem para que a DMS seja incluída no grupo de doenças neuromusculares que, eventualmente, impedem a contração normal do músculo estapédio, corroborando com os achados de outro estudo ${ }^{(18)}$.

Sendo a DMS uma doença progressiva, questionou-se a relação entre esses achados e a possibilidade de comprometimento do músculo estapédio. A hipótese levantada foi de que haja redução da força muscular desse músculo, o que pode gerar déficit na proteção da orelha interna, mais especificamente da cóclea e acarretar em perda auditiva neurossensorial. Nessa pesquisa, este fato foi observado por alteração no limiar das frequiências altas. Essa hipótese discorda de estudo anterior ${ }^{(16)}$, já que para este, a alteração do músculo estapediano ocasiona perda auditiva mais acentuada nas freqüências baixas.

Ainda, em relação ao reflexo estapediano, o efeito da elevação de limiares desse reflexo em $20 \%$ das orelhas, nas freqüências de $0,5,1$ e $2 \mathrm{kHz}$, coincide com diferentes trabalhos $^{(5-7,18)}$, no que tange à possibilidade de ocorrência de fenômeno miotônico no músculo estapediano, que acarretaria aumento do tempo de relaxamento muscular, proporcionando níveis de reflexo elevados.

Esses achados correlacionados com o declínio do reflexo estapediano, histórico otológico negativo e ausência de incompatibilidade entre audiometria tonal e vocal, podem sugerir alterações como: hiperacusia e/ou alteração do processamento auditivo, relacionados a uma possível alteração retrococlear.

Em relação ao efeito de anti-mascaramento, observouse ausência do reflexo estapediano na frequiência de $4 \mathrm{kHz}$, limiares auditivos normais, declínio do reflexo acústico nas freqüências de 0,5 e $1 \mathrm{kHz}$ e relato de dificuldade na compreensão auditiva em situação de diálogo em $20 \%$ da população estudada. Supõe-se que, esse percentual, sem histórico otológico prévio, seja sugestivo de comprometimento do complexo olivar superior, indicando possível alteração do processamento auditivo central, como descrito em outros estudos ${ }^{(11,16)}$.

Apenas $10 \%$ apresentaram curva timpanométrica do tipo C, 
divergindo de outros achados ${ }^{(4)}$, que não relataram alterações em curva timpanométrica em sujeitos com DMS. Esse dado que se refere ao $\mathrm{S} 5$ e a presença da curva timpanométrica do tipo $\mathrm{C}$, relaciona-se diretamente com o tipo da perda auditiva em orelha direita do paciente, conforme o Quadro 1.

Apesar de não ter sido encontrada nenhuma alteração da função da tuba auditiva, essa avaliação foi sugerida no intuito de investigar a ação do músculo tensor do palato, responsável pelo bom funcionamento da tuba auditiva, visto que estes sujeitos estão susceptíveis a apresentarem tal alteração pelo quadro muscular da doença.

Na literatura, não há estudos que forneçam a porcentagem de ocorrência do declínio do reflexo acústico em pacientes com DMS. No estudo em questão, apenas $10 \%$ dos indivíduos apresentaram declínio do reflexo acústico nas freqüências de 0,5 e $1 \mathrm{kHz}$. Hipotetizou-se que a ocorrência desse efeito, presente em limiares auditivos normais, sugere a fatigabilidade do músculo estapediano. Assim, a função do músculo pode estar comprometida pela fraqueza muscular presente na DMS.

Levantou-se a hipótese de que a presença de $10 \%$ com declínio do reflexo acústico nas frequiências de 0,5 e 1 kHz, em limiares auditivos normais, poderiam indicar lesões de tronco encefálico alto ou baixo, sugerida por outro estudo ${ }^{(12)}$. Mais especificamente essa lesão poderia estar localizada no complexo olivar superior, visto que essa mesma porcentagem apresentou reflexos acústicos ausentes nas freqüências de 2 e $4 \mathrm{kHz}$.

A perda auditiva nas freqüências altas pode gerar prejuízos na comunicação desses indivíduos. Seria viável a realização de audiometria de altas freqüências, que poderia revelar perda neurossensorial ou outras alterações auditivas, antes que esta fique evidente a partir de $8 \mathrm{kHz}$. Assim, os prejuízos na inteligibilidade da fala poderiam ser minimizados.

Sugere-se, para futuras pesquisas, a mensuração do reflexo ipsilateral a fim de investigar possíveis lesões em tronco encefálico alto ou baixo, proposta em outro estudo ${ }^{(12)}$. Essa questão surgiu a partir da junção dos achados do reflexo estapediano e do seu declínio em limiares auditivos normais.

Ressalta-se que avaliações mais criteriosas com a realização de tomografias computadorizadas e otoemissões acústicas, com efeitos de supressão, poderão esclarecer questões quanto a mal-formações do osso temporal e integridade das vias retrococleares, complementando a bateria audiológica de sujeitos portadores de DMS.

\section{CONCLUSÃO}

O estudo revelou que o padrão audiológico típico da distrofia de Steinert nesse grupo pesquisado foi de perda auditiva em frequiências altas, níveis de reflexo estapediano elevados ou ausentes, devido à presença de fraqueza muscular e possível ocorrência de fenômeno miotônico.

Ficou evidente na pesquisa que portadores de DMS que apresentarem reflexos estapedianos ausentes com limiares auditivos normais devem ser submetidos à avaliação do processamento auditivo central, principalmente aqueles que já apresentam dificuldade para entender conversas.

\begin{abstract}
Purpose: To identify the main hearing disorders in individuals with Steinert's myotonic dystrophy. Methods: Five individuals were submitted to tonal and vocal audiometry, acoustic impedance tests, examination of Eustaquian tube function, acoustic reflex investigation, and acoustic reflex decay. They also answered two questionnaires: a) Performance of auditory function; b) Psycho-acoustic-social investigation. Results: The study showed that $60 \%$ of the sample presented normal hearing thresholds, $10 \%$ had conductive hearing loss and 30\% had hearing loss in high frequencies. Concerning acoustic reflexes, it was observed that $30 \%$ of the subjects presented deficits at 0.5 and $1 \mathrm{kHz}$ and $40 \%$ at 2 and $4 \mathrm{kHz}$. Acoustic reflex decay was observed in $10 \%$ of the sample at 0,5 and $1 \mathrm{kHz}$. Conclusion: The study showed that the typical auditory profile of high-frequencies hearing loss was observed in $30 \%$ of the sample.
\end{abstract}

Keywords: Myotonic dystrophy/physiopathology; Myotonic dystrophy/complications; Hearing disorders; Hearing; Acoustic impedance tests; Stapedius; Eustachian tube; Reflex/genetics

\title{
REFERÊNCIAS
}

1. Fonseca LF. Compêndio de neurologia infantil. Rio de Janeiro: Editora Médica e Científica; 2002.

2. Rowland LP. Merrit tratado de neurologia. $10^{\mathrm{a}}$ ed. Rio de Janeiro: Guanabara Koogan; 2002.

3. Chiappetta AL, Odaz AL, Zanoteli E, Guilherme A, Oliveira AS. Disfagia orofaríngea na distrofia miotônica: avaliação fonoaudiológica e análise nasofibrolaringoscópica. Arq Neuropsiquiatr. 2001;59(2B):394400 .

4. Huygen PL, Verhagen WI, Noten JF. Auditory abnormalities, including 'precocious presbyacusis', in myotonic dystrophy. Audiology. 1994;33(2):73-84.
5. Osanai R, Kinoshita M, Hirose K. Stapedial reflex in myotonic dystrophy type 1 and CTG repeat expansion. J Neurol. 2001;248(12):1056-61.

6. Smirne S, Cesarani A, Franceschi M. Risultati preliminari sul comportamento del riflesso stapediale nella distrofia miotonica. Riv Neurol. 1976; 46:460-5.

7. Zakrisson JE, Blom S. The relaxation time of the stapedius reflex in dystrophia myotonica (Steinert). Acta Otolaryngol. 1982;94(5-6):4737.

8. Borg E, Zakrisson JE. The activity of the stapedius muscle in man during vocalization. Acta Otolaryngol. 1975;79(5-6):325-33. 
9. Meneguello J, Domenico ML, Costa MC, Leonhardt FD, Barbosa LH, Pereira LD. Ocorrência de reflexo acústico alterado em desordens do processamento auditivo. Rev Bras Otorrinolaringol. 2001; 67(6):8305 .

10. Carvalho RM, Soares JC. Efeito do estímulo facilitador no limiar de reflexo acústico. Rev Bras Otorrinolaringol. 2004; 70(2):200-6.

11. Ferreira LP; Befi-Lopes, DM, Limong SC. Tratado de fonoaudiologia. São Paulo: Roca; 2004.

12. Marotta RM, Quintero SM, Marone SA. Avaliação do processamento auditivo por meio do teste de reconhecimento de dissílabos em tarefa dicótica SSW em indivíduos com audição normal e ausência do reflexo acústico contralateral. Rev Bras Otorrinolaringol. 2002; 68(2):254-61.
13. Wright RB, Glantz RH, Butcher J. Hearing loss in myotonic dystrophy. Ann Neurol. 1988;23(2):202-3.

14. Lassaletta L, Fernández-Zubilaga A, González T, Gavilán J. Internal auditory canal hyperostosis in myotonic dystrophy. Otol Neurotol. 2005;26(2):310-1.

15. Jerger J, Oliver TA, Chmiel RA. Prediction of dynamic range from stapedius reflex in cochlear implant patients. Ear Hear. 1988;9(1):4-8.

16. Katz J. Tratado de audiologia clínica. São Paulo: Manole; 1999.

17. Jerger J. Clinical experience with impedance audiometry. Arch Otolaryngol. 1970;92(4):311-24.

18. Musiek FE, Rintelmann WF. Perspectivas atuais em avaliação auditiva. São Paulo: Manole; 2001.

\section{Anexo 1. Anamnese}

\section{Identificação}

Nome:

DN: Idade: Sexo: ( ) M ( ) F

Endereço:

Telefones para contato:

Anamnese

Q.1. Doenças metabólicas

( ) Sim ( ) Não

\section{Q.2. Doenças infecciosas}

( ) Sim ( ) Não

\section{Q.3. Tabagismo}

( ) Sim ( ) Não

\section{Q.4. Alcoolismo}

( ) Sim ( ) Não

Q.5. Faz uso de alguma medicação controlada?

( ) Sim Especificar:

Q.6. História de DMS na família?

( ) Sim ( ) Não Qual o grau de parentesco?

Q.7. Sente algum tipo de fraqueza no músculo?

( ) Não ( ) Sim ( ) mm inferiores

( ) $\mathrm{mm}$ superiores

( ) $\mathrm{mm}$ facial e/ou cervical

( ) $\mathrm{mm}$ da região abdominal

( ) $\mathrm{mm}$ da região torácica

Q.8. Quando foi diagnosticada a doença?

( ) 01 ano ( ) 01 a 05 anos ( ) mais de 05 anos 
Anexo 2. Protocolo de desempenho da função auditiva

Q.1. Queixa auditiva

( ) Sim ( ) Não

Q.2. Já realizou algum exame auditivo?

( ) Não ( ) Sim (responder Q.15)

Q.3. Há quanto tempo? ( ) mais de 6 meses

( ) menos de 6 meses (responder Q.16)

Q.4. Tem otalgia? ( ) Não

( ) $\operatorname{Sim}($ ) OD ( ) OE

Q.5. Tem otorréia? ( ) Não

( ) Sim ( ) OD ( ) Límpida ( ) Purulenta ( ) Sanguinolenta

( ) OE ( ) Límpida ( ) Purulenta ( ) Sanguinolenta

Q.6. Tem prurido? ( ) Não

( ) Sim ( ) OD ( ) OE

Q.7. Tem histórico de otites? ( ) Não

$$
\text { ( ) } \operatorname{Sim}(\text { ) OD ( ) OE }
$$

Q.8. Tem antecedentes familiares de perda auditiva?

( ) Sim ( ) Não

Q.9. Já realizou alguma cirurgia? ( ) Não

( ) Sim ( ) OD ( ) OE

Q.10. Já sofreu algum TCE?

( ) Sim ( ) Não

Q.11. Já sofreu algum trauma acústico (explosões, estampidos de armas de fogo, estúdios de gravação musical, indústrias)?
( ) Não
( ) $\operatorname{Sim}($ ) OD ( ) OE

Q.12. Utiliza ou utilizou algum medicamento por tempo prolongado (estreptomicina, canamicina, neomicina gestamicina viomicina cisplatina)?

( ) $\operatorname{Sim}($ ) Não
Anexo 3. Investigação psico-acústica-social

Q.1. Acha que escuta bem?

( ) $\operatorname{Sim}($ ) Não

Q.2. Tem zumbido?

( ) Não ( ) Sim ( ) agudo

( ) grave

Q.3. Sente tontura ou vertigem?

( ) Sim ( ) Não

Q.4. Tem plenitude aural?

( ) Não ( ) Sim ( ) OD

( ) OE

Q.5. Tem incômodo a sons fortes?

( ) Não ( ) Sim ( ) OD

( ) OE

Q.6. Ouve TV e rádio em forte intensidade?

( ) Sim ( ) Não

Q.7. Tem dificuldade para entender conversa?

( ) Sim ( ) Não

Q.8. Já trabalhou em ambiente ruidoso?

( ) $\operatorname{Sim}$ ( ) Não

Q.9. Sente desconforto a sons de alta intensidade?

( ) Sim ( ) Não

Q.10. Tem dificuldade ao falar ao telefone?

( ) Sim ( ) Não 